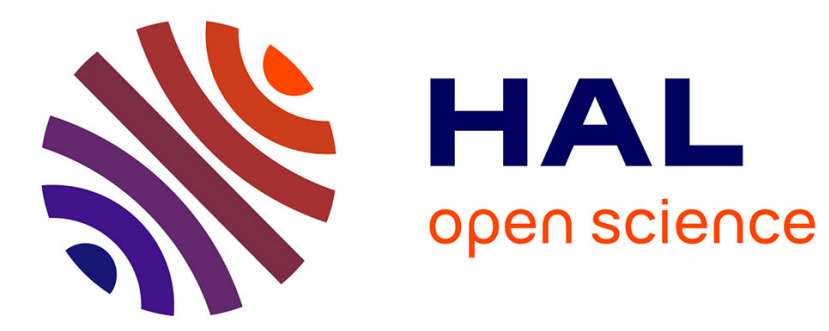

\title{
Measurement of Hospital Efficiency, Using a Latent Class Stochastic Frontier Model
}

Carlos Pestana Barros, António Gomes Menezes, José António Cabral Vieira

\section{To cite this version:}

Carlos Pestana Barros, António Gomes Menezes, José António Cabral Vieira. Measurement of Hospital Efficiency, Using a Latent Class Stochastic Frontier Model. Applied Economics, 2011, 45 (01), pp.4754. 10.1080/00036846.2011.579061 . hal-00732115

\section{HAL Id: hal-00732115 https://hal.science/hal-00732115}

Submitted on 14 Sep 2012

HAL is a multi-disciplinary open access archive for the deposit and dissemination of scientific research documents, whether they are published or not. The documents may come from teaching and research institutions in France or abroad, or from public or private research centers.
L'archive ouverte pluridisciplinaire HAL, est destinée au dépôt et à la diffusion de documents scientifiques de niveau recherche, publiés ou non, émanant des établissements d'enseignement et de recherche français ou étrangers, des laboratoires publics ou privés. 


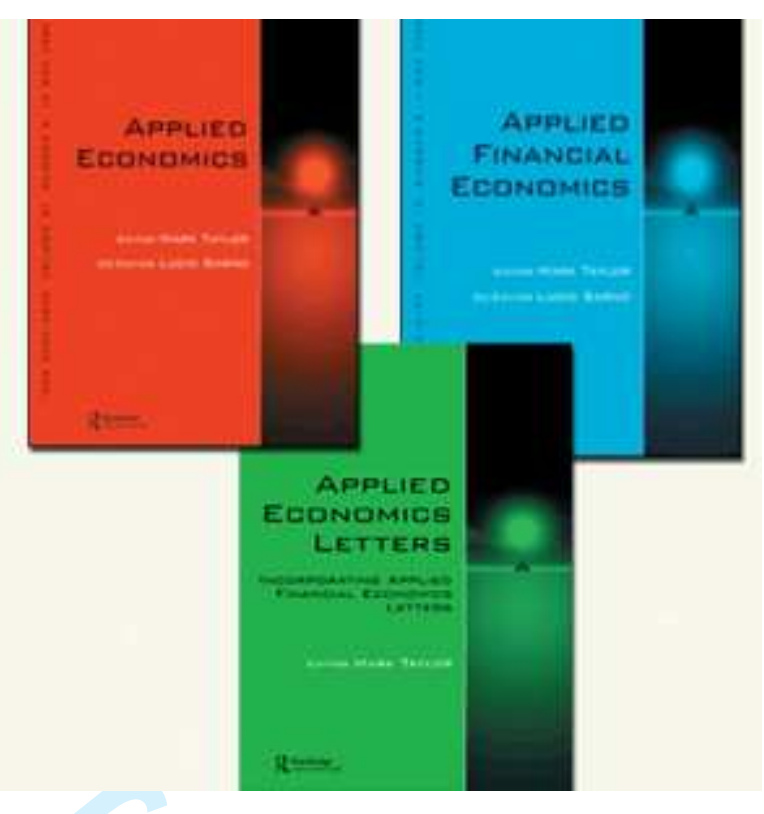

\section{Measurement of Hospital Efficiency, Using a Latent Class Stochastic Frontier Model}

\begin{tabular}{|c|c|}
\hline Journal: & Applied Economics \\
\hline Manuscript ID: & APE-08-0288.R1 \\
\hline Journal Selection: & Applied Economics \\
\hline $\begin{array}{r}\text { Date Submitted by the } \\
\text { Author: }\end{array}$ & 15-Aug-2010 \\
\hline Complete List of Authors: & $\begin{array}{l}\text { Barros, Carlos; Technical University of Lisbon, Economics } \\
\text { Menezes, António; University of the Azores, Economics } \\
\text { Vieira, José António; University of the Azores, Economics }\end{array}$ \\
\hline JEL Code: & $\begin{array}{l}\text { I10 - General < I1 - Health < I - Health, Education, and Welfare, } \\
\text { C29 - Other < C2 - Econometric Methods: Single Equation Models < } \\
\text { C - Mathematical and Quantitative Methods }\end{array}$ \\
\hline Keywords: & $\begin{array}{l}\text { Hospitals, random frontier, Translog frontier model, Decision- } \\
\text { making }\end{array}$ \\
\hline
\end{tabular}

\section{SCHOLARONE \\ Manuscripts}




\title{
Measurement of Hospital Efficiency, Using a Latent Class Stochastic Frontier Model
}

\author{
Carlos Pestana Barros ${ }^{\mathrm{a}}$ António Gomes de Menezes ${ }^{\mathrm{b}}$ and José Cabral Vieira ${ }^{\mathrm{b}}$ \\ a Instituto Superior de Economia e Gestão (ISEG), Technical University of Lisbon, \\ Portugal. E-mail: Cbarros@iseg.utl.pt \\ ${ }^{\mathrm{b}}$ University of Azores and CEEAplA
}

\begin{abstract}
This paper analyses the technical efficiency of Portuguese hospitals from 1997 to 2008 with the latent class frontier model, enabling the identification of different segments in the cost frontier. It is found that there are three statistically significant segments in the sample, leading to the conclusion that no common health policy can be applicable to all of the hospitals analysed, calling rather for policies conceived for each hospital segment identified. The health policy based in the identified segments enables a more accurate and cost-effective management of resources.
\end{abstract}

Key words: Hospitals, stochastic cost frontier, latent class model, technical efficiency, panel data.

JEL-code: I1, C29

\section{Introduction}

Efficiency in hospitals is a theme that has attracted much research in recent decades (Friesner; Rosemanand McPherson, 2007; Barbeta, Turati and Zago, 2007; Staat, 2006; Steinmann and Zweifel, 2003; Prior, 1996; Maniadakis and Thanassoulis, 2000; Parkin and Hollingsworth, 1997). A review of the literature shows two main approaches: the DEA - data envelopment analysis (Ozcan, Luke and Haksever, 1987; Burgess and Wilson, 1993; Dalmau and Puig-Junoy, 1998; Siciliani, 2006); and the 
stochastic frontier models (Barbeta, Turati and Zago, 2007; Siciliani, 2006; Blank and Eggink, 2004; Greene, 2004; Rosko, 2001).

The aim of the present study goes beyond previous research by adopting the latent frontier model, in line with Orea and Kumbhakar (2004). The work's objective is to combine operational and financial variables in the evaluation of hospital efficiency (Skinner, 1994). The distinctions between these groups of factors - operational and financial - means that merging the two constitutes a technical problem that requires the support of econometric techniques, such as frontier models. The motivation for the present research is based on the fact that hospitals in Portugal can be identified as having either public, private, or mixed status. The aim of this paper is to investigate the statistical factors that characterise the hospitals, clustering the sample in segments enabling us to define specific health policies for identified segments and to monitor each group with adequate targets. This procedure permits a more focused, cost-effective health policy, which can be generalised and applied to samples other than that for Portugal.

The contribution of this paper within hospital research is twofold. First, it adopts an innovative stochastic frontier model, which endogenously identifies segments of hospitals in a sample, overcoming the hypothesis of homogeneity of the variables adopted by the frontier models mentioned earlier. Secondly, it applies the model to Portuguese hospitals. Given that there is rigorous regulation of hospital services, this paper uses an innovative model that may improve our understanding of the best way to regulate the service as a whole (Hadley and Zuckerman, 1994).

The paper is organised as follows: Section 2, explains the method; Section 3 presents the data; Section 4 presents the results; Section 5 discusses the results and makes the concluding remarks. 


\section{Latent Class Frontier Models}

In this paper, we adopt the stochastic cost econometric frontier approach (Kumbhakar and Lovell, 2000). The general frontier cost function proposed by Aigner et al. (1977) and Meeusen and van den Broeck (1977) is the following:

$$
C_{i t}=C\left(X_{i t}\right) \cdot e^{v_{i t}+u_{i t}} ; \mathrm{i}=1,2, \ldots \mathrm{N}, \mathrm{t}=1,2, \ldots T
$$

Where $C_{i t}$ represents a scalar cost of the decision-unit $i$ under analysis in the $t$-th period; $X_{i t}$ is a vector of variables including the input prices and the output descriptors present in the cost function; and $\varepsilon_{i t}=u_{i t}+v_{i t}$ is the error term. This term may be decomposed into two components:

(i) the error term $v_{i t}$ is the one that is traditional of the econometric models, assumed to be independently and identically distributed, that represents the effect of random shocks (noise $v_{i t} \sim N\left(0, \sigma_{v}^{2}\right)$ ) and is independent of $u_{i t}$;

(ii) the inefficient term $u_{i t}$ represents technical inefficiencies and is assumed to be positive and normally distributed with a zero mean and variance $\sigma_{u}^{2}$. The positive disturbance $u_{i t}$ is reflected in a half-normal independent distribution truncated at zero, signifying that each hospital's cost must lie on or above its cost frontier. This implies that any deviation from the frontier is caused by management factors controlled by the hospitals.

The total variance is defined as $\sigma^{2}=\sigma_{v}^{2}+\sigma_{u}^{2}$. The contribution of the error term to the total variation is as follows: $\sigma_{v}^{2}=\sigma^{2} /\left(1+\lambda^{2}\right)$. The contribution of the inefficient term is: $\sigma_{u}^{2}=\sigma^{2} \cdot \lambda^{2} /\left(1+\lambda^{2}\right)$, where $\lambda$ is defined as $\lambda=\frac{\sigma_{u}}{\sigma_{v}}$, providing an indication of the relative contribution of $u$ and $v$ to $\varepsilon=u+v$. 
Given that the estimation procedures of equation (1) yield merely the residual $\varepsilon$, rather than the inefficiency term $u$, this term in the model must be calculated indirectly (Greene, 2000). In the case of panel data, such as that used in this paper, Battese and Coelli (1988) use the conditional expectation of $u_{i t}$, conditioned on the realised value of the error term $\varepsilon_{i t}=\left(v_{i t}+u_{i t}\right)$, as an estimator of $u_{i t}$. In other words, $E\left[u_{i t} / \varepsilon_{i t}\right]$ is the mean productive inefficiency for the $i$-th hospital at any time " $t$ ". Following Orea and Kumbhakar (2004), we can write equation (1) as a latent class frontier model:

$$
\left.C_{i t}\right|_{j}=\left.C\left(X_{i t}\right)\right|_{j} \cdot e^{\left.v_{i t}\right|_{j}+\left.u_{i t}\right|_{j}} ; \mathrm{i}=1,2, \ldots \mathrm{N}, \mathrm{t}=1,2, \ldots T
$$

Where subscript " $i$ " denotes the hospital, " $t$ " indicates time and " $j$ " represents the different classes or groups. The vertical bar signifies that there is a different model for each class " $j "$ and, therefore, each hospital belongs to the same group in all the periods. Assuming that $v$ is normally distributed and $u$ follows a half-normal distribution, the likelihood function $(L F)$ for each hospital company " $i$ " at time " $t$ " for group " $j$ " is (Cf. Greene, 2004):

$$
L F_{i j}=C\left(x_{i t}, \beta_{j}, \sigma_{j}, \lambda_{j}\right)=\frac{\Phi\left(\left.\lambda_{j} \cdot \varepsilon_{i t}\right|_{j} / \sigma_{j}\right)}{\Phi(0)} \cdot \frac{1}{\sigma_{j}} \cdot \phi\left(\frac{\left.\varepsilon_{i t}\right|_{j}}{\sigma_{j}}\right)
$$

Where $\left.\varepsilon_{i t}\right|_{j}=\left.\ln C_{i t}\right|_{j}-\beta_{j}^{\prime} x_{i t}, \quad \sigma_{j}=\left[\sigma_{u j}^{2}+\sigma_{v j}^{2}\right]^{1 / 2}, \lambda_{j}=\sigma_{u j} / \sigma_{v j}$, while $\phi$ denotes the standard normal density and $\Phi$ the cumulative distribution function. The likelihood function for hospital " $i$ " in group " $j$ ” is obtained as the product of the likelihood functions in each period. See Orea and Kumbhakar (2004) for details. 
3. Data

The dataset was compiled from the Portuguese Ministry of Health records and from the financial accounts of the hospitals. Our dataset covers the years from 1997 to 2008 and 51 hospitals, i.e., the vast majority of hospitals in Portugal. In order to have hospitals with similar natures and technology, this sample includes all the district hospitals.

Table 1 presents the characteristics of the variables used in the analysis. We transformed the variables according to the description column. We adopted the traditional log-log specification (Translog) to allow for the possible non-linearity of the frontier. We specify a translog variable cost frontier model with capital as a quasi-fixed factor (Caves and Christiensen, 1980; Kaparakis et al., 1994).

Table 1: Descriptive Statistics of the Data 1997-2008

\begin{tabular}{|c|c|c|c|c|c|}
\hline Variable & Description & Minimum & Maximum & Mean & $\begin{array}{l}\text { Standard } \\
\text { deviation }\end{array}$ \\
\hline $\operatorname{logVCT}$ & $\begin{array}{l}\text { Log of variable cost at constant price } \\
2002\end{array}$ & 15.714 & 19.342 & 18.132 & 0.712 \\
\hline Trend & Trend variable & 1 & 12 & 6.5 & 3.293 \\
\hline $\log Y 1$ & $\begin{array}{l}\text { Output measured by number of } \\
\text { discharged patients }\end{array}$ & 7.745 & 10.538 & 9.342 & 0.574 \\
\hline $\log Y 2$ & $\begin{array}{l}\text { Intermediate input measured by the } \\
\text { length of stay in hospital }\end{array}$ & 1.410 & 2.895 & 2.152 & 0.195 \\
\hline $\log Y 3$ & $\begin{array}{l}\text { Output variable measured by the log } \\
\text { of external consultations }\end{array}$ & 9.507 & 12.557 & 11.052 & 0.639 \\
\hline $\log \mathrm{Y} 4$ & $\begin{array}{l}\text { Output variable measured by log of } \\
\text { emergency visits }\end{array}$ & -2.302 & 12.290 & 11.052 & 1.840 \\
\hline Log Mix & $\begin{array}{l}\text { Intermediate input measured by the } \\
\text { mix of specialist services }\end{array}$ & -0.289 & 0.335 & 0.006 & 0.090 \\
\hline $\log K$ & $\begin{array}{l}\text { Capital proxied by the number of } \\
\text { beds }\end{array}$ & -0.157 & 0.059 & -0.052 & 0.072 \\
\hline Log W1 & $\begin{array}{l}\text { Price of labour measured by the ratio } \\
\text { of wages by the number of } \\
\text { employees }\end{array}$ & 8.579 & 10.410 & 10.017 & 0.188 \\
\hline Log W2 & $\begin{array}{l}\text { Price of other factors proxied by the } \\
\text { regional price index }\end{array}$ & 4.189 & 6.493 & 5.537 & 0.568 \\
\hline RAA & Dummy variable equal to one for & 0 & 1 & 0.125 & 0.33112 \\
\hline
\end{tabular}




\begin{tabular}{|c|c|c|c|c|c|}
\hline & $\begin{array}{l}\text { hospitals located on Azores islands } \\
\text { and zero otherwise }\end{array}$ & & & & \\
\hline SA & $\begin{array}{l}\text { Dummy variable equal to one for } \\
\text { hospitals with private enterprise } \\
\text { status and zero otherwise }\end{array}$ & 0 & 1 & 0.421 & 0.231 \\
\hline DivK & $\begin{array}{l}\text { Dummy equal to one for hospitals } \\
\text { serving more than one county and } \\
\text { zero otherwise }\end{array}$ & 0 & 1 & 0.2745 & 0.4468 \\
\hline Quality & $\begin{array}{l}\text { Dummy equal to one for hospitals } \\
\text { with management system credited by } \\
\text { the King's Fund and zero otherwise }\end{array}$ & 0 & 1 & 0.632 & 0.271 \\
\hline
\end{tabular}

\section{Results}

The empirical specification of the cost function is the translog. We have chosen a flexible functional form in order to avoid imposing unnecessary a priori restrictions on the technologies to be estimated. Each explanatory variable is divided by its geometric mean. In this way, the translog can be considered as an approximation to an unknown function and the first-order coefficients can be interpreted as the production elasticities evaluated at the sample geometric mean. We also include both a time trend and a squared time trend in order to obtain some temporal changes (Breyer, 1987). The equation to be estimated is:

$$
\begin{aligned}
& \log V C T_{i t}=\left.\beta_{0}\right|_{j}+\left.\beta_{y}\right|_{j} \log Y_{i t}+\left.\sum_{l} \beta_{w l}\right|_{j} \log W_{i l t}+\left.\frac{1}{2} \beta_{y y}\right|_{j} \log \left(Y_{i t}\right)^{2}+ \\
& \left.\frac{1}{2} \sum_{l} \beta_{w w l}\right|_{j} \log W_{i l t}^{2}+\left.\sum_{l} \beta_{w l y}\right|_{j} \log W_{i l t} \log Y_{i t}+\left.\delta_{t}\right|_{j} \cdot t+\left.\delta_{t t}\right|_{j} \cdot t^{2}+\left.v_{i t}\right|_{j}+\left.u_{i}\right|_{j}
\end{aligned}
$$

where VCT is the variable cost of the hospital, $Y$ is the output, $W$ denotes input price, $t$ is a time trend, $v$ is a random error which reflects the statistical noise and is assumed to follow a normal distribution centered at zero, while $u$ reflects inefficiency and is assumed to follow a half-normal distribution. In order to test the number of groups, we used the testing-down procedure proposed by Greene (2005), in which beginning from a $J^{*}$ known to be at least as large as the true $J$, one can test down, given that the $J$ - 1 class model is nested with the $J$ class model imposing $\theta_{\mathrm{j}}=\theta_{\mathrm{j}-1}$, based on likelihood ratio tests, as well as the SBIC and AIC. The model with four groups does not converge, likewise 
Table 2: Latent Translog Panel Production Frontier (dependent variable: $\log$ VCT)

\begin{tabular}{|c|c|c|c|c|}
\hline Variables & Standard SF. & Latent class 1 & Latent class 2 & Latent class 2 \\
\hline & $\begin{array}{l}\text { Coefficients } \\
\text { (t-ratio) }\end{array}$ & $\begin{array}{l}\text { Coefficients } \\
\text { (t-ratio) }\end{array}$ & $\begin{array}{l}\text { Coefficients } \\
\text { (t-ratio) }\end{array}$ & $\begin{array}{l}\text { Coefficients } \\
\text { (t-ratio) }\end{array}$ \\
\hline Constant & $\begin{array}{c}-2.123 \\
(-1.321)^{*}\end{array}$ & $\begin{array}{c}-2.132 \\
(-2.999) * * *\end{array}$ & $\begin{array}{c}-1.321 \\
(3.219)^{* * *}\end{array}$ & $\begin{array}{c}-1.129 \\
(-2.932)^{* * *}\end{array}$ \\
\hline Trend & $\begin{array}{c}0.901 \\
(3.212)^{*}\end{array}$ & $\begin{array}{c}0.115 \\
(3.138)^{* * *}\end{array}$ & $\begin{array}{c}0.109 \\
(3.893)^{* * * *}\end{array}$ & $\begin{array}{c}0.112 \\
(3.892)^{* * *}\end{array}$ \\
\hline $\log Y 1$ & $\begin{array}{c}0.731 \\
(2.423)^{* *}\end{array}$ & $\begin{array}{c}1.544 \\
(2.324)^{* *}\end{array}$ & $\begin{array}{c}0.125 \\
(3.512)^{* * *}\end{array}$ & $\begin{array}{c}0.217 \\
(4.124)^{* * *}\end{array}$ \\
\hline $\log Y 2$ & $\begin{array}{l}0.128 * \\
(1.027)\end{array}$ & $\begin{array}{c}1.718 \\
(2.453)^{* *}\end{array}$ & $\begin{array}{c}0.727 \\
(4.329)^{* * *}\end{array}$ & $\begin{array}{c}0.529 \\
(3.128)^{* * *}\end{array}$ \\
\hline $\log Y 3$ & $\begin{array}{c}0.425 \\
(3.123)^{* * *}\end{array}$ & $\begin{array}{c}0.812 \\
(3.318) * * *\end{array}$ & $\begin{array}{c}0.216 \\
(3.124)^{* * *}\end{array}$ & $\begin{array}{c}0.135 \\
(3.137)^{* * *}\end{array}$ \\
\hline $\log \mathrm{Y} 4$ & $\begin{array}{c}0.065 \\
(0.043)\end{array}$ & $\begin{array}{c}0.071 \\
(3.219)^{* * *}\end{array}$ & $\begin{array}{c}0.231 \\
(3.018)^{* * *}\end{array}$ & $\begin{array}{c}0.531 \\
(3.218) * * *\end{array}$ \\
\hline Log Mix & $\begin{array}{c}-0.038 \\
(-0.071)\end{array}$ & $\begin{array}{c}-1.802 \\
(-2.045) * *\end{array}$ & $\begin{array}{c}-0.128 \\
(-3.162)^{* * *}\end{array}$ & $\begin{array}{c}-0.381 \\
(-4.117) * * *\end{array}$ \\
\hline $\log K$ & $\begin{array}{c}0.171 \\
(0.183)\end{array}$ & $\begin{array}{c}1.075 \\
(2.173)^{* *}\end{array}$ & $\begin{array}{c}1.012 \\
(3.421)^{* * *}\end{array}$ & $\begin{array}{c}0.923 \\
(3.219) * * *\end{array}$ \\
\hline $\log W 1$ & $\begin{array}{c}0.042 \\
(0.152)\end{array}$ & $\begin{array}{c}0.827 \\
(3.189)^{* * *}\end{array}$ & $\begin{array}{c}0.129 \\
(4.129)^{* * *}\end{array}$ & $\begin{array}{c}0.327 \\
(4.215)^{* * *}\end{array}$ \\
\hline Log W2 & $\begin{array}{c}0.045 \\
(0.071) \\
\end{array}$ & $\begin{array}{c}0.319 \\
(3.132)^{* * *} \\
\end{array}$ & $\begin{array}{c}0.271 \\
(4.219)^{* * *}\end{array}$ & $\begin{array}{c}0.283 \\
(3.419) * * *\end{array}$ \\
\hline Trend $^{2}$ & $\begin{array}{c}-0.181 \\
(-0.038)\end{array}$ & $\begin{array}{c}-0.563 \\
(-2.532)^{* *}\end{array}$ & $\begin{array}{c}-0.215 \\
(-4.219)^{* * *}\end{array}$ & $\begin{array}{c}-0.215 \\
(-3.128) * * *\end{array}$ \\
\hline $1 / 2 \log (Y 1)^{2}$ & $\begin{array}{c}0.017 \\
(3.002)^{* * *}\end{array}$ & $\begin{array}{c}1.272 \\
(2.201)^{* *}\end{array}$ & $\begin{array}{c}0.579 \\
(2.626)^{* *}\end{array}$ & $\begin{array}{c}0.832 \\
(2.835)^{* *}\end{array}$ \\
\hline $1 / 2 \log (Y 2)^{2}$ & $\begin{array}{c}0.731 \\
(2.590)^{* *}\end{array}$ & $\begin{array}{c}0.153 \\
(0.945)\end{array}$ & $\begin{array}{c}1.076 \\
(3.229)^{* * *}\end{array}$ & $\begin{array}{c}0.534 \\
(1.674)^{*}\end{array}$ \\
\hline $1 / 2 \log (Y 3)^{2}$ & $\begin{array}{c}0.131 \\
(4.218)^{* * *}\end{array}$ & $\begin{array}{c}0.082 \\
(0.820)\end{array}$ & $\begin{array}{c}0.021 \\
(4.126)^{* * *}\end{array}$ & $\begin{array}{c}0.032 \\
(2.784)^{* *}\end{array}$ \\
\hline $1 / 2 \log (Y 4)^{2}$ & $\begin{array}{c}0.189 \\
(4.324)^{* * *}\end{array}$ & $\begin{array}{c}0.185 \\
(3.568)^{* * *}\end{array}$ & $\begin{array}{c}0.257 \\
(3.783)^{* * *}\end{array}$ & $\begin{array}{c}0.217 \\
(3.219)^{* * *}\end{array}$ \\
\hline
\end{tabular}

\footnotetext{
${ }^{1}$ The program used to estimate the model was Limdep 9.0.
} 


\begin{tabular}{|c|c|c|c|c|}
\hline 1/2Log $\operatorname{Mix}^{2}$ & $\begin{array}{c}0.328 \\
(1.832)^{*}\end{array}$ & $\begin{array}{c}0.210 \\
(2.674)^{* *}\end{array}$ & $\begin{array}{c}0.315 \\
(3.067)^{* * *}\end{array}$ & $\begin{array}{c}0.878 \\
(2.197)^{* *}\end{array}$ \\
\hline $1 / 2 \log K^{2}$ & $\begin{array}{c}0.319 \\
(4.027)^{* * *}\end{array}$ & $\begin{array}{c}0.128 \\
(3.534)^{* * *}\end{array}$ & $\begin{array}{c}0.128 \\
(3.710)^{* * *}\end{array}$ & $\begin{array}{c}0.068 \\
(3.419)^{* * *}\end{array}$ \\
\hline $1 / 2 \log (\mathrm{W} 1)^{2}$ & $\begin{array}{c}0.021 \\
(1.074)\end{array}$ & $\begin{array}{c}0.278 \\
(1.452)\end{array}$ & $\begin{array}{c}0.629 \\
(3.058)^{* * *}\end{array}$ & $\begin{array}{c}0.420 \\
(3.129)^{* * *}\end{array}$ \\
\hline $1 / 2 \log (\mathrm{W} 2)^{2}$ & $\begin{array}{c}0.031 \\
(0.308)\end{array}$ & $\begin{array}{c}0.216 \\
(1.145)^{*}\end{array}$ & $\begin{array}{c}0.814 \\
(3.457)^{* * *}\end{array}$ & $\begin{array}{c}0.791 \\
(2.967) * * *\end{array}$ \\
\hline $\log Y 1 * \log Y 2$ & $\begin{array}{c}1.217 \\
(3.015)^{* * *}\end{array}$ & $\begin{array}{c}1.278 \\
(2.378)^{* *}\end{array}$ & $\begin{array}{c}0.279 \\
(3.073)^{* * *}\end{array}$ & $\begin{array}{c}0.126 \\
(4.219)^{* * *}\end{array}$ \\
\hline $\log Y 1 * \log Y 3$ & $\begin{array}{c}0.113 \\
(3.427)^{* * *}\end{array}$ & $\begin{array}{c}1.012 \\
(2.342)^{* *}\end{array}$ & $\begin{array}{c}0.167 \\
(0.110)\end{array}$ & $\begin{array}{c}0.719 \\
(1.210)\end{array}$ \\
\hline $\log Y 1^{*} \log Y 4$ & $\begin{array}{c}0.821 \\
(4.295)^{* * *}\end{array}$ & $\begin{array}{c}0.218 \\
(0.452)\end{array}$ & $\begin{array}{c}0.290 \\
(2.278)^{* *}\end{array}$ & $\begin{array}{c}0.781 \\
(3.189)^{* * *}\end{array}$ \\
\hline LogY1* Log Mix & $\begin{array}{c}0.318 \\
(3.045)^{* * *}\end{array}$ & $\begin{array}{c}0.829 \\
(1.002)\end{array}$ & $\begin{array}{c}0.428 \\
(3.531)^{* * *}\end{array}$ & $\begin{array}{c}0.523 \\
(2.054)^{*}\end{array}$ \\
\hline $\log \mathrm{Y}^{*} \log \mathrm{K}$ & $\begin{array}{c}0.329 \\
(3.145)^{* * *}\end{array}$ & $\begin{array}{c}-0.016 \\
(-0.119)\end{array}$ & $\begin{array}{c}-0.135 \\
(-3.228)^{* * *}\end{array}$ & $\begin{array}{l}-0.153 \\
(1.078)\end{array}$ \\
\hline $\log \mathrm{Y} 1 * \log \mathrm{W} 1$ & $\begin{array}{c}-0.348 \\
(-3.319)^{* * *}\end{array}$ & $\begin{array}{c}-0.568 \\
(-1.945)^{*}\end{array}$ & $\begin{array}{c}-0.174 \\
(-3.219) * * *\end{array}$ & $\begin{array}{c}-0.114 \\
(2.978) * * *\end{array}$ \\
\hline LogY1* Log W2 & $\begin{array}{c}-0.078 \\
(-3.045)^{* * *}\end{array}$ & $\begin{array}{c}-0.057 \\
(-0.726)\end{array}$ & $\begin{array}{c}-0.109 \\
(-3.805) * * *\end{array}$ & $\begin{array}{c}-0.120 \\
(-2.317)\end{array}$ \\
\hline $\log Y 2 * \log Y 3$ & $\begin{array}{c}-0.135 \\
(-3.110)^{* * *}\end{array}$ & $\begin{array}{c}-0.129 \\
(-0.341) \\
\end{array}$ & $\begin{array}{c}-0.251 \\
(-3.672)^{* * *}\end{array}$ & $\begin{array}{c}-0.239 \\
(-2.534)^{* *}\end{array}$ \\
\hline $\log Y 2 * \log Y 4$ & $\begin{array}{c}0.267 \\
(3.729)^{* * *}\end{array}$ & $\begin{array}{c}0.254 \\
(0.371)\end{array}$ & $\begin{array}{c}0.332 \\
(4.512)^{* * *}\end{array}$ & $\begin{array}{c}0.612 \\
(1.674)^{* * *}\end{array}$ \\
\hline LogY2* Log Mix & $\begin{array}{c}0.137 \\
(3.218)^{* * *}\end{array}$ & $\begin{array}{c}0.818 \\
(2.224)^{* *}\end{array}$ & $\begin{array}{c}0.151 \\
(2.321)^{* * *}\end{array}$ & $\begin{array}{c}0.528 \\
(3.017) * * *\end{array}$ \\
\hline $\log Y 2 * \log K$ & $\begin{array}{c}0.042 \\
(5212)^{* * *}\end{array}$ & $\begin{array}{c}0.351 \\
(1.432)\end{array}$ & $\begin{array}{c}0.185 \\
(3.162)^{* * *}\end{array}$ & $\begin{array}{c}0.312 \\
(3.072)^{* * *}\end{array}$ \\
\hline $\log Y 2 * \log \mathrm{W} 1$ & $\begin{array}{c}0.038 \\
(4.345)^{* * *}\end{array}$ & $\begin{array}{c}0.167 \\
(0.032) \\
\end{array}$ & $\begin{array}{c}0.282 \\
(2.412)^{* * *}\end{array}$ & $\begin{array}{c}0.305 \\
(3.127)^{* * *}\end{array}$ \\
\hline $\log Y 2 * \log \mathrm{W} 2$ & $\begin{array}{c}-0.067 \\
(-2.623) \\
\end{array}$ & $\begin{array}{l}-0.621 \\
(0.778) \\
\end{array}$ & $\begin{array}{c}-0.296 \\
(-4.029) * * *\end{array}$ & $\begin{array}{c}-0.104 \\
(-1.029) * * *\end{array}$ \\
\hline Log Y3* LogY4 & $\begin{array}{c}-0.363 \\
(-3.195)^{* * *}\end{array}$ & $\begin{array}{c}-1.578 \\
(-1.710)^{* *}\end{array}$ & $\begin{array}{c}0.271 \\
(3.619) * * *\end{array}$ & $\begin{array}{c}0.219 \\
(3.395) * * *\end{array}$ \\
\hline LogY3* Log Mix & $\begin{array}{c}-0.073 \\
(-3.174)^{* * * *}\end{array}$ & $\begin{array}{c}-0.191 \\
(-1.029)\end{array}$ & $\begin{array}{c}-0.471 \\
(-1.712)^{* *}\end{array}$ & $\begin{array}{c}-0.325 \\
(-1.106)^{*}\end{array}$ \\
\hline LogY3* Log K & $\begin{array}{c}0.845 \\
(1.219) \\
\end{array}$ & $\begin{array}{c}0.110 \\
(0.135)\end{array}$ & $\begin{array}{c}0.151 \\
(3.128)^{* * *}\end{array}$ & $\begin{array}{c}0.517 \\
(3.029)^{* * *}\end{array}$ \\
\hline LogY3* $\log \mathrm{W} 1$ & $\begin{array}{c}-0.347 \\
(-2.753)^{* *}\end{array}$ & $\begin{array}{c}-0.289 \\
(-1.232)^{* *}\end{array}$ & $\begin{array}{c}-0.715 \\
(-1.218)^{*}\end{array}$ & $\begin{array}{c}-0.612 \\
(-1.292)^{*}\end{array}$ \\
\hline LogY3* $\log \mathrm{W} 2$ & $\begin{array}{c}0.583 \\
(3.819)^{* * *}\end{array}$ & $\begin{array}{c}-0.779 \\
(-1.682)^{* *}\end{array}$ & $\begin{array}{c}-0.821 \\
(-0.321) \\
\end{array}$ & $\begin{array}{c}-0.419 \\
(-1.157) \\
\end{array}$ \\
\hline LogY4* Log Mix & $\begin{array}{c}0.371 \\
(3.045)^{* * *}\end{array}$ & $\begin{array}{c}0.141 \\
(1.305)^{*}\end{array}$ & $\begin{array}{c}0.579 \\
(2.626)^{* *}\end{array}$ & $\begin{array}{c}0.525 \\
(2.012)^{*}\end{array}$ \\
\hline $\log \mathrm{Y}^{*} * \log \mathrm{K}$ & $\begin{array}{c}0.256 \\
(3.356)^{* * *}\end{array}$ & $\begin{array}{c}0.135 \\
(1.674)^{*}\end{array}$ & $\begin{array}{c}1.076 \\
(2.229)^{* *}\end{array}$ & $\begin{array}{c}0.725 \\
(3.126)^{* * *}\end{array}$ \\
\hline LogY4* $\log \mathrm{W} 1$ & $\begin{array}{c}-0.178 \\
(-3.162)^{* * *}\end{array}$ & $\begin{array}{c}-0.187 \\
(-0.359)\end{array}$ & $\begin{array}{c}-0.021 \\
(-0.126)\end{array}$ & $\begin{array}{c}-0.115 \\
(-1.123)\end{array}$ \\
\hline
\end{tabular}




\begin{tabular}{|c|c|c|c|c|}
\hline Log $\mathrm{Y}^{*} * \log \mathrm{W} 2$ & $\begin{array}{c}0.801 \\
(3.132)^{* * *}\end{array}$ & $\begin{array}{c}1.89 \\
(2.128)^{* *} \\
\end{array}$ & $\begin{array}{c}0.810 \\
(2.925)^{* *}\end{array}$ & $\begin{array}{c}0.612 \\
(2.729)^{* *} \\
\end{array}$ \\
\hline $\log \operatorname{Mix}^{*} \log \mathrm{K}$ & $\begin{array}{c}0.127 \\
(4.129) * * *\end{array}$ & $\begin{array}{c}0.321 \\
(0.712)\end{array}$ & $\begin{array}{c}0.282 \\
(3.031)^{* * *}\end{array}$ & $\begin{array}{c}0.229 \\
(3.110)^{* * *}\end{array}$ \\
\hline Log Mix*log W1 & $\begin{array}{c}-0.187 \\
(-4.028)^{* * *}\end{array}$ & $\begin{array}{c}-1.032 \\
(-1.256)\end{array}$ & $\begin{array}{c}-0.620 \\
(-3.721)^{* * *}\end{array}$ & $\begin{array}{c}-0.819 \\
(-3.524) * * *\end{array}$ \\
\hline Log Mix*Log W2 & $\begin{array}{c}0.219 \\
(1.828)^{* *}\end{array}$ & $\begin{array}{c}0.253 \\
(2.023)^{* *}\end{array}$ & $\begin{array}{c}0.307 \\
(3.291)^{* * *}\end{array}$ & $\begin{array}{c}0.810 \\
(3.190)^{* * * *}\end{array}$ \\
\hline $\log \mathrm{K}^{*} \log \mathrm{W} 1$ & $\begin{array}{c}0.178 \\
(4.032)^{* * *}\end{array}$ & $\begin{array}{c}0.823 \\
(4.219)^{* * *}\end{array}$ & $\begin{array}{c}0.871 \\
(3.510)^{* * *}\end{array}$ & $\begin{array}{c}0.226 \\
(3.131)^{* * *}\end{array}$ \\
\hline $\log \mathrm{K}^{*} \log \mathrm{W} 2$ & $\begin{array}{c}0.017 \\
(1.292)^{*}\end{array}$ & $\begin{array}{c}0.045 \\
(3.129)^{* * * *}\end{array}$ & $\begin{array}{c}0.028 \\
(3.731)^{* * *}\end{array}$ & $\begin{array}{c}0.126 \\
(3.056)\end{array}$ \\
\hline $\log \mathrm{W} 1 * \log \mathrm{W} 2$ & $\begin{array}{c}0.018 \\
(0.219)\end{array}$ & $\begin{array}{c}0.032 \\
(0.178)\end{array}$ & $\begin{array}{c}0.051 \\
(0.029)\end{array}$ & $\begin{array}{c}0.118 \\
(1.319)\end{array}$ \\
\hline RAA & $\begin{array}{c}0.527 \\
(2.318)^{* *}\end{array}$ & $\begin{array}{c}0.719 \\
(2.521)^{* *}\end{array}$ & $\begin{array}{c}0.538 \\
(1.819)^{*}\end{array}$ & $\begin{array}{c}0.228 \\
(1.418)^{*}\end{array}$ \\
\hline SA & $\begin{array}{c}-1.062 \\
(-2.128)^{* *}\end{array}$ & $\begin{array}{c}-0.631 \\
(-2.219)^{* *}\end{array}$ & $\begin{array}{c}-0.243 \\
(-3.184) * * *\end{array}$ & $\begin{array}{c}-0.220 \\
(-3.719) * * *\end{array}$ \\
\hline DivK & $\begin{array}{c}-0.031 \\
(-0.052)\end{array}$ & $\begin{array}{c}-0.045 \\
(-0.372)\end{array}$ & $\begin{array}{c}-0.027 \\
(-1.290)\end{array}$ & $\begin{array}{c}0.156 \\
(1.219) *\end{array}$ \\
\hline Quality & $\begin{array}{c}0.232 \\
(3.073)^{* * *}\end{array}$ & $\begin{array}{c}-0.175 \\
(2.210)^{* *}\end{array}$ & $\begin{array}{c}0.517 \\
(4.289)^{* * *}\end{array}$ & $\begin{array}{c}0.475 \\
(3.087)^{* * *}\end{array}$ \\
\hline$\sigma=\left(\sigma_{v}^{2}+\sigma_{u}^{2}\right)^{l / 2}$ & 0.412 & $0.271 * *$ & $0.317 *$ & $0.325^{*}$ \\
\hline$\lambda=\sigma_{u} / \sigma_{v}$ & 140.316 & 240.98 & 175.31 & 234.02 \\
\hline Log Likelihood Function & 111 & 115 & & \\
\hline NOBS & 612 & 612 & 612 & 612 \\
\hline
\end{tabular}

(t-statistics) in parentheses are below the parameters. $*=$ significance at $10 \%, * *=5 \%$, and $* * *=1 \%$.

The variables have the expected signs, since all price elasticities are positive. Moreover, instead of imposing homogeneity on prices, we have tested for it and accept that all segments have homogeneity in prices. It can be seen that the group with the highest labour elasticity is the first. The costs increase with the trend, but at a decreasing rate for all groups. Another interesting outcome is that the estimated coefficients of the standard stochastic frontier are, in general, between the minimum and the maximum of the latent class model coefficients. This result suggests that to some extent, the standard stochastic frontier estimates an average of the latent class model technologies.

\subsection{Efficiency Groups}

First we present the groups formed by the latent class model using the posterior probabilities of class membership in Table 3. 
Table 3: Group Composition

\begin{tabular}{|c|c|c|c|}
\hline & Group 1 & Group 2 & Group 3 \\
\hline 1 & $\mathrm{CH}$ Torres Vedras & H Santa Maria da Feira & H Infante D. Pedro - Aveiro \\
\hline 2 & H Divino Espirito Santo & H Sao Bernardo - Setubal & H Dr. Francisco Zagalo - Ovar \\
\hline 3 & H Mirandela & H Faro & $\mathrm{CH}$ Coimbra \\
\hline 4 & CH Alto Minho & CH P. Varzim V. Conde & H Garcia de Orta - Almada \\
\hline 5 & H Sousa Martins - Guarda & CH Vila Nova de Gaia & H Montijo \\
\hline 6 & CH Caldas da Rainha & H Santa Luzia - Elvas & H Chaves \\
\hline 7 & H Santo Tirso & H Espirito Santo - Evora & H Egas Moniz \\
\hline 8 & CH Cascais & H Curry Cabral & H Santo Espirito de Angra do Heroismo \\
\hline 9 & CH Barlavento Algarvio & H S. Francisco Xavier & H Pulido Valente \\
\hline 10 & H Braga & $\mathrm{CH}$ Cova da Beira & H Horta \\
\hline 11 & H Padre Americo - Vale do Sousa & H Lamego & H Sao Teotonio - Viseu \\
\hline 12 & H Reinaldo dos Santos - Vila Franca de Xira & H Sao Joao da Madeira & H Santa Maria Maior de Barcelos \\
\hline 13 & H Sao Miguel - Oliveira de Azemeis & H Jose Maria Grande - Portalegre & CH Medio Tejo \\
\hline 14 & H Braganca & H Sao Joao de Deus - Famalicao & H Agueda \\
\hline 15 & CH Baixo Alentejo & H Sao Goncalo - Amarante & H Santarem \\
\hline 16 & H Figueira da Foz & H Amato Lusitano - Castelo Branco & H Senhora da Oliveira - Guimaraes \\
\hline 17 & H Santo Andre - Leiria & H Nossa Senhora do Rosario - Barreiro & CH Vila Real Peso da Regua \\
\hline Observations & 204 & 204 & 204 \\
\hline
\end{tabular}

We observe that the most efficient hospitals appear in the first group. The second group consists of hospitals with an average level of efficiency. Finally, the third group comprises hospitals with the lowest levels of efficiency.

Finally, Table 4 shows the average cost efficiency for each hospital in the period. The cost efficiency is defined as the ratio between the minimum cost and the actual cost and takes values between 0 and 1 . According to this definition, the closer to 1 is the efficiency measure, the more efficient must the hospital be considered. Given that the dependent variable is expressed in logarithms, it was calculated as:

$$
E C=\exp (-\hat{a})
$$

where the estimated value of the inefficiency $(\hat{u})$ is separated from the random error term ( $(\hat{v})$ using the Jondrow et al. (1982) formula.

Table 4: Average Cost Efficiency in the Period

$\begin{array}{llccc}\text { Nobs } & \text { Hospitals } & \text { Standard SF } & \begin{array}{c}\text { Latent Class } \\ \text { Model }\end{array} & \begin{array}{c}\text { Difference } \\ 1\end{array} \\ \text { CH Vila Real Peso da Regua } & 0.56 & 0.66 & 0.10 \\ 2 & \text { H Senhora da Oliveira - Guimaraes } & 0.55 & 0.67 & 0.12 \\ 3 & \text { H Santarem } & 0.62 & 0.68 & 0.06 \\ 4 & \text { H Agueda } & 0.58 & 0.69 & 0.11\end{array}$




\begin{tabular}{|c|c|c|c|c|}
\hline 5 & CH Medio Tejo & 0.59 & 0.70 & 0.11 \\
\hline 6 & H Santa Maria Maior de Barcelos & 0.60 & 0.72 & 0.12 \\
\hline 7 & H Sao Teotonio - Viseu & 0.62 & 0.73 & 0.11 \\
\hline 8 & H Horta & 0.65 & 0.74 & 0.09 \\
\hline 9 & H Pulido Valente & 0.67 & 0.75 & 0.08 \\
\hline 10 & H Santo Espirito de Angra do Heroismo & 0.67 & 0.76 & 0.09 \\
\hline 11 & H Egas Moniz & 0.68 & 0.77 & 0.09 \\
\hline 12 & H Chaves & 0.69 & 0.78 & 0.09 \\
\hline 13 & H Montijo & 0.70 & 0.79 & 0.09 \\
\hline 14 & H Garcia de Orta - Almada & 0.70 & 0.80 & 0.10 \\
\hline 15 & CH Coimbra & 0.70 & 0.82 & 0.12 \\
\hline 16 & H Dr. Francisco Zagalo - Ovar & 0.71 & 0.83 & 0.12 \\
\hline 17 & H Infante D. Pedro - Aveiro & 0.72 & 0.84 & 0.12 \\
\hline 18 & H Nossa Senhora do Rosario - Barreiro & 0.73 & 0.84 & 0.11 \\
\hline 19 & H Amato Lusitano - Castelo Branco & 0.74 & 0.85 & 0.11 \\
\hline 20 & H Sao Goncalo - Amarante & 0.75 & 0.85 & 0.10 \\
\hline 21 & H Sao Joao de Deus - Famalicao & 0.76 & 0.86 & 0.10 \\
\hline 22 & H Jose Maria Grande - Portalegre & 0.77 & 0.86 & 0.09 \\
\hline 23 & H Sao Joao da Madeira & 0.78 & 0.86 & 0.08 \\
\hline 24 & H Lamego & 0.79 & 0.87 & 0.08 \\
\hline 25 & CH Cova da Beira & 0.79 & 0.87 & 0.08 \\
\hline 26 & H S. Francisco Xavier & 0.80 & 0.88 & 0.08 \\
\hline 27 & H Santo Andre - Leiria & 0.81 & 0.89 & 0.08 \\
\hline 28 & H Curry Cabral & 0.83 & 0.90 & 0.07 \\
\hline 29 & H Espirito Santo - Evora & 0.84 & 0.90 & 0.06 \\
\hline 30 & H Santa Luzia - Elvas & 0.85 & 0.91 & 0.06 \\
\hline 31 & CH Vila Nova de Gaia & 0.86 & 0.92 & 0.06 \\
\hline 32 & CH P. Varzim V. Conde & 0.87 & 0.92 & 0.05 \\
\hline 33 & H Faro & 0.88 & 0.93 & 0.05 \\
\hline 34 & H Sao Bernardo - Setubal & 0.89 & 0.94 & 0.05 \\
\hline 35 & H Santa Maria da Feira & 0.89 & 0.94 & 0.05 \\
\hline 36 & H Braganca & 0.90 & 0.95 & 0.05 \\
\hline 37 & H Sao Miguel - Oliveira de Azemeis & 0.90 & 0.95 & 0.05 \\
\hline 38 & H Reinaldo dos Santos - Vila Franca de Xira & 0.91 & 0.95 & 0.04 \\
\hline 39 & H Padre Americo - Vale do Sousa & 0.91 & 0.96 & 0.05 \\
\hline 40 & H Braga & 0.91 & 0.96 & 0.05 \\
\hline 41 & CH Barlavento Algarvio & 0.92 & 0.96 & 0.04 \\
\hline 42 & H Figueira da Foz & 0.93 & 0.97 & 0.04 \\
\hline 43 & $\mathrm{CH}$ Cascais & 0.94 & 0.98 & 0.04 \\
\hline 44 & H Santo Tirso & 0.95 & 0.98 & 0.03 \\
\hline 45 & CH Caldas da Rainha & 0.96 & 0.98 & 0.02 \\
\hline 46 & H Sousa Martins - Guarda & 0.97 & 0.98 & 0.01 \\
\hline 47 & CH Alto Minho & 0.98 & 0.99 & 0.01 \\
\hline 48 & H Mirandela & 0.99 & 0.99 & 0.00 \\
\hline 49 & H Divino Espirito Santo & 0.99 & 0.99 & 0.00 \\
\hline 50 & CH Torres Vedras & 0.99 & 0.99 & 0.00 \\
\hline 51 & CH Baixo Alentejo & 1.00 & 1.00 & 0.00 \\
\hline & Mean & 0.799 & 0.868 & 0.068 \\
\hline & Median & 0.800 & 0.880 & 0.080 \\
\hline & Std. Dev & 0.131 & 0.100 & 0.036 \\
\hline
\end{tabular}


The hospitals with the lowest cost efficiencies are $\mathrm{CH}$ Vila Real Peso da Regua and $\mathrm{H}$ Senhora da Oliveira - Guimarães. It should be emphasised that these two hospitals are located in small towns in the north of mainland Portugal. The highest cost-efficiency scores are achieved by $\mathrm{CH}$ Baxo Alentejo, $\mathrm{CH}$ Torres Vedras, H Divino Espirito Santo and H Mirandela.

Concerning the comparison between the standard stochastic frontier and the latent class model efficiencies, it is worth indicating that the rankings are preserved and the latent frontier displays greater cost efficiency for all hospitals. The average difference is 0.036, which is rather significant. Likewise, the differences are not consistent for all hospitals, implying that the latent class model efficiencies are not merely a constant added to the standard stochastic frontier model.

\section{Discussion and Conclusion}

The interpretation of the obtained results is as follows. First, they allow us to conclude that latent frontier models describe the Portuguese hospitals well, when allowing for heterogeneity and defining segments in the sample. This is possibly the most significant finding of the present paper. The implication of this result is that a common government policy would be inappropriate for all hospitals, since the three segments identified point to the existence of heterogeneity. Therefore, any health policy which involves some of these heterogeneous variables should be tailored according to the segments. Second, our analysis has led us to identify three segments. However, this does not mean that there are only three segments to be found in the Portuguese hospital network (Dismuke and Sena, 2004). Rather, it signifies that given the small number of observations available, the model cannot distinguish more segments. The third finding is that hospitals in the first segment are defined as generic Portuguese hospitals, a 
picture that emerges with traditional homogenous frontier models (Barros et al. 2008). These hospitals are characterised by the outputs and inputs increasing the costs, which have a positive relationship with $R A A$ and a negative relationship with $S A, D i v K$ and quality, signifying that these variables decrease costs. However, DivK is not statistically significant. The results for the first segment are intuitive and in accordance with the economic theory (Cf. Varian, 1987).

In the second segment, the hospitals' costs have the same relationship with outputs, inputs and input prices. Moreover, the dummy variables have the same relationship with costs that was observed in the first segment, with the exception of quality, which is now positive. These are hospitals that have adopted the quality standards, but do not maintain them. The third segment is distinct from the other two, based on the negative sign of DivK, i.e., hospitals serving more than one county, which is now positive and statistically significant.

What is the overall rationale for these results? They are in fact quite intuitive, since hospitals are not homogenous. For instance, there are small and large hospitals in the sample. These tangible characteristics result in different performance levels, dissimilar flux and discrepancies in their financial debts, which account for the different segments in the sample. In the case of the Portuguese hospitals, there are three different segments that can be distinguished on the grounds of the values and signs of the exogenous variables.

With regard to the implications of the results, first, there is evidence that private hospitals (SA) are more efficient than public hospitals, since SA characteristics decrease costs. This outcome is based on the negative sign of the attributes of private hospitals. This result is also supported in previous research in this field, with alternative models. This result serves as a cross-validation of previous results in this field. 
Second, the sign of credit hospitals in all segments (quality) is negative in the first segment, but positive in the other two. Therefore, we conclude that credit hospitals, motivated by the quest for quality, perform efficiently (Williamson, 1979, 1985). However, those hospitals that do not maintain the quality procedures resort to using them as a device to conceal bad managerial practices.

Third, based on the sign of the variable DivK in all segments, hospitals serving more than one county perform efficiently, while seeking enhanced performance (Caves and Porter, 1977).

Finally, given the sign of the variable $R A A$ in all segments, the mainland hospitals seeking profits perform efficiently (Barney, 1991; Rumelt, 1991).

Hence, it can be concluded that, in this context, private hospitals are more efficient than public hospitals. This is supported by the theory of transaction costs and property rights (Klein, Crawford and Alchian, 1978; Williamson, 1979, 1985), signifying that Portuguese hospitals allocate dedicated asset-specificity (Williamson, 1985) according to the population density.

Therefore, assuming the asset-specificity strategy, hospitals managed by whichever contracts are assumed to be efficient, displaying unique specific management assets, are seen as exhibiting inherently differentiated levels of efficiency and sustainable production, which are ultimately a return on the unique assets owned and controlled by the hospitals (Teece et al., 1997). The property resource is the main factor segmenting the sample. In addition, the strategic-groups theory (Caves and Porter, 1977), which justifies different efficiency scores on the grounds of differences in the structural characteristics of units within an industry or sector, could partially explain the differences observed in the efficiency of Portuguese hospitals. All these dynamics are 
derived from a clear allocative contract that minimises transaction costs and property rights (Klein, Crawford and Alchian, 1978; Williamson, 1979, 1985).

\section{References}

Aigner, D.J., Lovell, C.A.K. and Schmidt, P. (1977). Formulation and estimation of stochastic frontier production function models, Journal of Econometrics, 6, 21-37.

Barbeta, G.P.; Turati, G. and Zago, A.M. (2007). Behavioural Differences between Public and Private Non-Profit Hospitals in the Italian Health Service. Health Economics, 16,1,75-96

Barney, J. (1991). Firm resources and sustained competitive advantage, Journal of Management, 17, 99-120.

Barros, C.P.; Menezes, A.G.; Peypoch, N.; Solonandrasana, B. and Vieira, J.C. (2008) $\mathrm{Na}$ analysis of hospital efficiency and productivity growth using the Luenberger indicator. Health Care Management Science, 11,4,373-381

Battese, G.E. and Coelli, T.J. (1988). Prediction of Firm-level technical efficiencies with a generalised frontier production function and panel data. Journal of Econometrics, 38, 387-399.

Blank, J.L.T. and Eggink, E. 2004. The Decomposition of Cost Efficiency: An Empirical Application of the Shadow Cost Function Model to Dutch General Hospitals. Health Care Management Science, 7,2, 79-88.

Breyer, F. (1987) The specification of a hospital cost function. The Review of Economics and Statistics, 79, 3, 443-453.

Burgess, J.F. and Wilson, D.W. 1983. Technical Efficiency in Veterans Administration Hospitals. In Fried, H.O.; Lovell, C.A.K. and Schmidt, S.S. (eds) The Measurement of Productivity Efficiency: Techniques and Applications. Oxford, Oxford University Press, 335-351. 
Caves R. and Porter M.E. (1977). From entry barriers to mobility barriers: Conjectural decisions and contrived deterrence to new competition. Quarterly Journal of Economics 91, 241-261.

Caves, D.W. and Christensen, L.R. (1980). The Relative Efficiency of Public and Private Firms in a Competitive Environment: The Case of Canadian Railroads". The Journal of Political Economy, 88,5, 958-976.

Dalmau, E. and Puig-Junoy 1998. Market Structure and Hospital Efficiency: Evaluating Potential Effects of Deregulation in National Health Service. Review of Industrial Organization, 13, 447-466.

Dismuke, C.E. and Sena, V. 2004. Has DRG payment influenced the technical Efficiency and Produtivity of Diagnostic Technologies in Portugal Public Hospitals: An Empirical Analysis using parametric and non-parametric methods. Health Care

Friesner, D.; Rosemanand, R. and McPherson, M.Q. (2007) Are_hospitals seasonally inefficient? Evidence from Washington State. Applied Economics, 40, 6,, Pages 699 723

Greene W. (2000). Econometric Analysis (4 ${ }^{\text {th }}$ ed.); London, Prentice Hall

Greene, W. (2005). Fixed and random effects in stochastic frontier models, Journal of Productivity Analysis, Vol. 23, pp. 7-32.

Greene, W. (2004). Distinguishing between heterogeneity and efficiency: stochastic frontier analysis of the World Health Organisation's panel data on national health care systems. Health Economics, 13, 959-980.

Hadley, J. and Zuckerman, S. (1994) The role of efficiency measurement in hospital rate setting. Journal of Health Economics, 13, 335-340. 
Klein, B.; Crawford, R.C. and Alchian, A. (1978) Vertical Integration, Appropriable Rents, and the Competitive Contracting Process. Journal of Law and Economics, 22, 297-326.

Maniadakis, N. and Thanassoulis,E. (2000). Assessing productivity changes in UK hospital reflecting technology and input price. Applied Economics, 32,12, 1575 1589

Meeusen, W. and van den Broeck, J. (1977). Efficiency estimation from a CobbDouglas production function with composed error. International Economic Review, 18, 435-444.

Orea, L., and Kumbhakar, S. (2004). Efficiency measurement using a latent class stochastic frontier model. Empirical Economics , 29, 169-183.

Ozcan, Y.A. and Luke, R.D. 1993. A national study of the efficiency of hospitals in urban markets. Health Services Research, February, 719-739.

Parkin, D. and Hollingsworth, B. (1997) Measuring production efficiency of acute hospitals in Scotland, 1991-94: validity issues in data envelopment analysis. Applied Economics, 29,11,1425 - 1433

Prior, D. (1996) Technical efficiency and scope economies in hospitals. Applied Economics, 28, 10, 1295 - 1301.

Rosko, M.D. 2001. Cost efficiency of US hospitals: a stochastic frontier approach. Health Economics, 10, 6: 539-551.

Rumelt, R. (1991). How much does industry matter? Strategic Management Journal, $12,2,167-185$.

Siciliani, L. 2006. Estimating technical Efficiency in the Hospital Sector with Panel Data: A Comparison of Parametric and Non-Parametric Techniques. Applied Health Economics \& Health Policy, 5,2, 99-116.

Skinner, J. (1994) What do stochastic frontier cost functions tell us about inefficiencies? Journal of Health Economics, 13, 323-328. 
Staat, M. (2006) Efficiency of hospital_in Germany: a DEA-bootstrap approach. Applied Economics, 38, Issue 19, 2255 - 2263

Steinmann, L. and Zweifel, P. (2003) On the (in)efficiency of Swiss hospitals. Applied Economics, 35, Issue 3, $361-370$

Teece D.; Pisano G. and Shuen A. (1997). Dynamic capabilities and strategic management. Strategic Management Journal, 18, 7, 509-533.

Varian, H.R. (1987). Intermediate Microeconomics: A Modern Approach. New York, W.W. Norton \& Co.

Williamson, O. (1981) The economics of organization: The transaction costs approach. American Journal of Sociology, 87, 548-577.

Williamson, O. (1985) The Economic Institutions of Capitalism: Firms, Markets and Vertical Contracting. Free Press, N.Y. 